\title{
Towards effective treatment of eating disorders: nothing is as practical as a good theory
}

Citation for published version (APA):

Jansen, A. T. M. (2001). Towards effective treatment of eating disorders: nothing is as practical as a good theory. Behaviour Research and Therapy, 39, 1007-1022. https://doi.org/10.1016/S0005-7967(01)00010-9

Document status and date:

Published: 01/01/2001

DOI:

10.1016/S0005-7967(01)00010-9

Document Version:

Publisher's PDF, also known as Version of record

Document license:

Taverne

\section{Please check the document version of this publication:}

- A submitted manuscript is the version of the article upon submission and before peer-review. There can be important differences between the submitted version and the official published version of record.

People interested in the research are advised to contact the author for the final version of the publication, or visit the DOI to the publisher's website.

- The final author version and the galley proof are versions of the publication after peer review.

- The final published version features the final layout of the paper including the volume, issue and page numbers.

Link to publication

\footnotetext{
General rights rights.

- You may freely distribute the URL identifying the publication in the public portal. please follow below link for the End User Agreement:

www.umlib.nl/taverne-license

Take down policy

If you believe that this document breaches copyright please contact us at:

repository@maastrichtuniversity.nl

providing details and we will investigate your claim.
}

Copyright and moral rights for the publications made accessible in the public portal are retained by the authors and/or other copyright owners and it is a condition of accessing publications that users recognise and abide by the legal requirements associated with these

- Users may download and print one copy of any publication from the public portal for the purpose of private study or research.

- You may not further distribute the material or use it for any profit-making activity or commercial gain

If the publication is distributed under the terms of Article $25 \mathrm{fa}$ of the Dutch Copyright Act, indicated by the "Taverne" license above, 


\title{
Towards effective treatment of eating disorders: nothing is as practical as a good theory
}

\author{
Anita Jansen \\ Maastricht University, Faculty of Psychology, Dept of Experimental Psychology, PO Box 616, 6200 MD, \\ Maastricht, The Netherlands
}

Accepted 17 January 2001

\begin{abstract}
There is much room for improvement in the treatment of eating disorders, anorexia nervosa in particular. It is argued that for more effective treatment a radical change in thinking and doing is needed. First, the wide-spread multicausal model of eating disorders must be abandoned and replaced by (a) fundamental strategic research into the most parsimonious explanation of eating disorders and (b) interventions solely directed on the specific maintaining mechanisms. Second, evidence-based working is needed in mental health care. In daily practice, two of three psychotherapists do not treat their eating disordered patients with the best treatment available, i.e. cognitive behaviour therapy. The Dutch Ministry of Health, Welfare and Sport tried to improve the care for eating disorder patients by the nomination of several specialist hospital units. These units are, however, not selected for their treatment quality or the use of evidencebased treatment protocols. It is argued that this ministerial operation will not increase the supply of effective treatment. The Minister obviously should have done two other things to improve the amount and quality of treatment supply for eating disorders: First, she better could invest in a broad array of workshops, training and supervision programs in cognitive behaviour therapy for all psychotherapists working with eating disorders. Second, since nothing is so practical as a good theory, the facilitation of research into parsimonious models of the relevant mechanisms as well as the experimental tests of interventions on these mechanisms would have been a promising move to effective treatment. (C) 2001 Elsevier Science Ltd. All rights reserved.
\end{abstract}

Keywords: Eating disorders; Policy; Evidence-based mental health

E-mail address: a.jansen@psychology.unimaas.nl (A. Jansen). 


\section{Introduction}

The Dutch Ministry of Health, Welfare and Sport has a special interest in eating disorders. The minister responsible (Mrs Borst) sees waiting lists as the primary bottleneck in the care for eating disordered patients. Eating disorders are in the Netherlands as common as in other Western cultures; more than 40,000 Dutch girls and young women (year-prevalence about 2\%) suffer from either Anorexia Nervosa or Bulimia Nervosa. The Binge Eating Disorder occurs in some $1 \%$ to $2 \%$ of the entire population, which amounts to 150,000 to 300,000 people in the Netherlands (SEN, 1998), whereas the exact incidence of the other eating disorders Not Otherwise Specified is not known. The severity of the disorders is substantiated by the figures on mortality: the number of patients who die of Anorexia Nervosa in the Netherlands and Germany is around 6\% of the anorexia cases (Van Hoeken \& Hoek, 1999; Fichter \& Quadflieg, 1999). In spite of all that, subjects with an eating disorder must wait an average of 3.6 months for clinical treatment, which can be too long in severe and life-threatening situations. For this reason, the Minister has nominated a number of general hospital units in the country as specialized in the treatment of eating disorders. In addition, she designated and subisidizes the eating disorder unit of a general psychiatric hospital for the treatment of particularly difficult and severe cases as well as for the coordination of the national treatment offer.

Employees at the Dutch Ministry of Health, Welfare and Sport as well as Dutch experts in the field reason that patients with eating disorders might benefit by the current nomination of these units. They suspect that, through this action of the Ministry, the waiting lists will decrease and treatment will be more effective. However, it might be a misconception that a mere nomination of several hospital units as being "specialised" in the treatment of eating disorders will decrease the waiting lists and increase the supply of effective treatment. One of the propositions of the present paper is that the Ministerial Order bears the hazard that the supply of effective treatment will only get smaller in the end.

Another central proposition is that nothing is as practical as a good theory. What will be clear later on is that the effectiveness of treatments for Anorexia Nervosa in particular is very slight. This is not at all surprising when considering the fact that there is still no explanation for why some people suffer or keep suffering from Anorexia Nervosa. In such a light, treatment quickly appears to be shooting with blanks and basically hit or miss. This is not a reproach of those providing treatment; they act to the best of their knowledge. But without a decent theory, they cannot get very far.

After a discussion of the alleged multicausal nature of eating disorders in the next section, a plea is made for doing more reductionistic experiments in order to identify the real factors that cause or maintain the disorders. It is argued that experimental intervention on the identified factors should decrease eating disorder symptoms and finally may provide for better treatment methods. These ideas are occasionally related to current practical knowledge and the policies of the Dutch government.

\section{On the origin and maintenance of eating disorders}

Eating disorders evolve according to typical patterns and can be more or less summarized in terms of three recurring characteristics: abnormal eating behaviour, a negative body image, and 
either successful or unsuccessful attempts to lose weight. In addition to the aforementioned specific eating pathology, people with eating disorders also suffer frequently from depression and anxiety (as a consequence of the eating disorder in most cases), personality disorders, and low self-esteem. Although thousands of articles on the topic of eating disorders appeared in scientific journals, it is still unknown how some people end up with an eating disorder.

The current lack of knowledge concerning the cause and maintenance of eating disorders has led many experts to argue - and actually believe — that eating disorders have an infinite number of causes. The multicausality model has been generally accepted and still figures as a most important "etiological model" in the eating disorders literature (Bloks, van Furth, \& Hoek, 1999; Brownell \& Fairburn, 1995; Garner, 1997; SEN, 1998; Vanderlinden, 2000). The model consists of nothing more and nothing less than a holistic matrix of the most abstract causal and maintenance factors. In such a manner, biological, psychological, and social levels of explanation are distinguished with a number of predisposing, eliciting, and maintenance factors surrounding these (see Fig. 1).

Advocates of the multicausal model emphasize the circular and idiosyncratic character of the possible interactions within this biopsychosocial entity. While the model makes a plausible impression at first glance, it is argued that the multicausal approach to eating disorders is an opportunistic solution that only produces ad hoc explanations. Further on, it is also considered why the multicausal model appears to enjoy such widespread popularity despite its obvious methodological shortcomings.

The multicausal model rests on the following assumptions: 1) that eating disorders emerge from a mix of factors that can differ per individual and 2) that the same factors and also the same mix of factors need not lead to an eating disorder in two different individuals (Garfinkel \& Garner, 1982, p. 191). These assumptions imply, which is very critical, that it is never possible to make a general statement regarding the emergence of eating disorders. Eating disorders are assumed to really have a changing spectrum of causes, and general laws to predict the occurrence or nonoccurrence of eating disorders are impossible to find. Such a model generates no hypotheses simply because it reflects, in the eyes of its creators, the ultimate reality. However, the belief that no

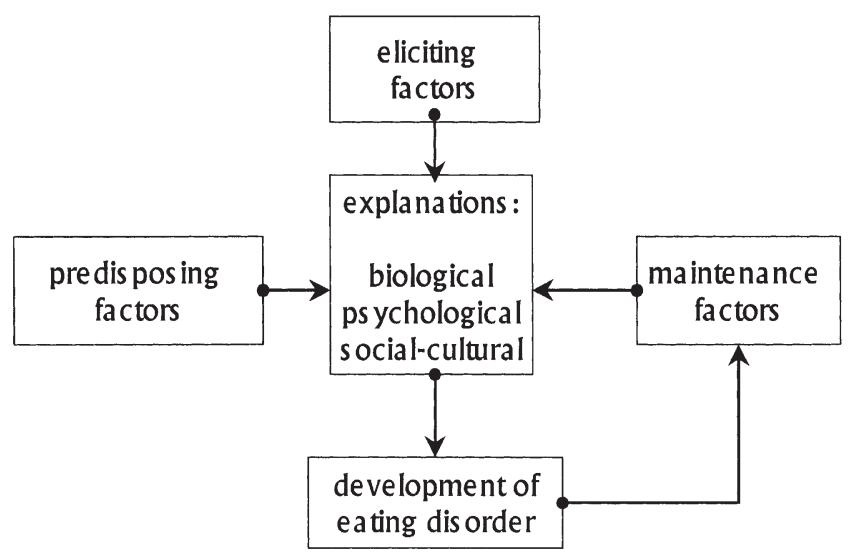

Fig. 1. The multicausal model of eating disorders. 
general laws underlie the emergence of eating disorders, and denial of a hypothesis-generating function for the model, places the multicausal model outside the domain of empirical science.

Prediction is the hallmark of empirical science. In the words of De Groot (1961): "If I know something, I can predict something; if I cannot predict anything, then I know nothing". From the multicausal model, little can be predicted. The model also forces researchers to turn in a direction other than the reductionist direction. Explanatory general mechanisms cannot be sought because the grounds for emergence are unique to each individual.

Growth of knowledge can catch up with the multifactorial manner of thinking (Jansen \& Boon, 1991). We saw this previously with AIDS. For a long time, it was assumed that AIDS had multiple causes. Until HIV was discovered: then it became clear that infection with HIV was the cause of AIDS. For this type of discovery, reduction and thus the experimental study of causal or maintening mechanisms is usually necessary. Only reduction can further help the patient in the end. It happens all too frequently and all too easily that research that is not concerned with the "total patient" is disposed of as an invalid ivory tower product. In such a manner, not only this pure form of research is discredited but also the development of sorely needed knowledge is blocked.

\section{Necessary and sufficient factors}

Which factors appear to be necessary and sufficient for the development of an eating disorder? Research into risk factors has been scant and, in the majority of cases, retrospective in nature. Fairburn discovered, by including a psychiatric control group in his research, that many of the suspected risk factors mentioned in the multicausal model do not specifically lead to eating disorders (Fairburn, Welch, Doll, Davies, \& O'Connor, 1997). Conversely, pre-morbid obesity weight, continued comments regarding bodily form, weight and eating behaviour from family members pre-morbid weight, and the eating behaviour of the patients appear — among other things - to play a specific role in the development of eating disorders. These factors prompt attempts to lose weight and thereby increase the risk of an eating disorder. This finding does not stand alone. Also Schwartz, Phares, Tantleff-Dunn, and Thompson (1999) found that women with eating disorders have been more frequently criticized for their bodily appearance during their youth than women without eating disorders.

These data appear to be important, but the research methods nevertheless call for some reserve. The aforementioned studies were retrospective in nature and self-report was the most important source of information. Retrospective self-report research into risk factors produces results with a potentially high degree of unreliability. Patients have a disorder and, at a time when they are clearly unhappy, they are asked about how things went earlier in their lives. How heavy were they then? What did others think of their appearance? It is conceivable that people who detest their own bodies and consider themselves fat even when they are emaciated may think that they always had such an elephant body and recall all kinds of teasing in retrospect.

A recently published prospective study of the determinants of dieting behaviour among schoolgoing children by Stice (Stice, Mazotti, Krebs, \& Martin, 1998) is methodologically neater and provides some critical results. These researchers found dissatisfaction with one's own body to be the best predictor by far of dieting behaviour. However, dissatisfaction with one's body was not 
strongly associated with actually being overweight or perceived pressure to be slender. In fact, heavier body weight and perceived pressure to be slender predicted dieting behaviour to only a very small extent or not at all. These findings suggest that the importance attached to thinness and a conviction of pre-morbid fatness may be a consequence of dieting rather than the reverse.

That satisfaction with one's body is not related to actual weight or perceived pressure to be slender (i.e., a thinness ideal) is an important insight. This finding also offers a great deal to think about and study for no matter how important research on risk factors may be, it says nothing about the emergence of eating disorders or the mechanisms that maintain such disorders. People tend to fall silent at this point when things actually start to get really exciting. How can some people perceive their body weight as being high when this is in fact not the case? Why are some people dissatisfied with their bodies when there is objectively nothing wrong? Is there really objectively nothing wrong? What is the exact nature of the dissatisfaction? How can dissatisfaction with one's body be reduced? Exactly these types of questions can be answered with experimental, reductionist research. Answering these questions is not only exciting but also useful. If we know how something fits together, then we are often able to fix it after it falls apart.

In our lab, the body image of a group women having a subclinical eating disorder was studied (Jansen, 2001a). What stood out was that the weight of the group with subclinical eating disorders was quite normal and higher but not significantly different from the weight of the normal control group, and that their waist-hip ratios were similar. As expected, the eating-disordered women evaluated their own bodies as much less attractive than the normal controls. What we did not expect was that a large forum of inhabitants from Maastricht $(n=72)$ also evaluated the bodies of the eating disordered subjects as less attractive, without knowing which subjects were eating disordered and which were not. It is more an academically interesting difference than a clinically important difference; the difference is small but significant. Just why the dieters were found to be less attractive is, of course, the next question and more reductionistic research concerned with this question is needed.

The data reviewed here suggest that dissatisfaction with one's body may be associated with a less attractive but certainly not too fat body. The dissatisfaction with one's body is mistakenly transferred to, projected onto, the weight. It is, after all, easier to do something about body weight than overly narrow shoulders, crooked knees, or short legs. When patients indeed know that they are not too fat, we have to do with illogical reasoning; "I am not too fat but ugly, so I should go on a diet". As if losing weight can help them get rid of something "ugly". Whether they really think that they are too fat must be answered not by asking them to answer questions (self-report) but by having them perform, for example, a cognitive task to demonstrate the logically-consistent but nevertheless incorrect reasoning. In other words, we are concerned with cognitive processes that may lie at the heart of the eating disorders. The cognitive processes can be precisely mapped via reductionist experiments. And as soon as a parsimonious model has been formulated, whether or not experimental manipulation of the processes considered responsible indeed elicits changes in the behaviour we aim to explain can be examined.

\section{Treatment of anorexia nervosa}

Despite the methodological shortcomings mentioned above, a multicausal vision is common among people working in the field. This popularity can be understood when it is postulated that 
the multicausal model rests on the generally unstated assumption that every person is unique. In clinical practice, the model gives clinicians the freedom to explain eating pathology in terms of all kinds of processes considered applicable to this unique patient.

An interesting question is whether strictly individual therapy is the logical consequence of a multicausal pathogenesis for eating disorders. Imagine that the eating disorder concerns a unique concrete event that cannot be explained in terms of general principles. Is it then the case that no general and empirical statements can be made about the treatment of the eating disorder? Most adherents to a multicausal approach consider a general solution indeed impossible. A simple medical example, however, shows this to be a clear misconception. Imagine two individuals both breaking their left leg but under completely different circumstances. Person A was running a marathon and slipped on an unevenness in the track. Person B came out of a bar in a drunken stupor and tripped over the curb. Persons A and B are very different. A is a sport person, has black curls, and looks through brown eyes at the world. Person B is a blond boor with sea blue eyes. Nevertheless, the medical recommendation in these two unique cases is the same: the leg in a cast. Apparently the exact cause of the broken leg (tripping over a curb or slipping on an unevenness in a track) and the individual characteristics of those involved (a dark sport man or a blond drunk) are not of importance for an empirical statement with regard to the best treatment. In the case of eating disorders as well, unfamiliarity with the exact cause of the condition is no reason for not making a statement about the best treatment. The treatment recommendation can always be subjected to empirical criteria; that is, without knowledge of the exact cause of the eating disorder, it is still possible to recommend the most successful form of therapy possible.

The usual treatment for Anorexia Nervosa is typically multifaceted. In addition to efforts to have the patient gain weight, she is also confronted with her own body in a mirror or on video. She must learn to talk about her emotions, receives psycho-education, creative therapy, dance therapy, interoceptive perception training, body-oriented therapy, improved self-esteem and social skills are worked on, and finally relapse prevention is also undertaken (Fichter \& Quadflieg, 1999; Bloks, van Furth, \& Hoek, 1999; Vanderlinden, 2000). German researchers have inventoried the effects of such broad spectrum or multimodal integral treatment (Fichter \& Quadflieg, 1999). It should be noted that the study certainly does not meet the methodological demands currently placed on outcome studies. There was no manipulation check, control groups were not used and nothing was standardized. Nevertheless, the German inventory provides us with some descriptive information. Exactly 103 patients with Anorexia Nervosa were admitted to a large medicalpsychological hospital for an average of 120 days and underwent the "broad spectrum treatment" described above. Six years following this treatment, the patients were studied using a wide range of measurements. Incidentally, 65\% of the patients received an average of 176 days of additional inpatient treatment for the eating disorder at another hospital. These patients thus had an average of 296 days of inpatient treatment behind them, which is a total of almost 10 months. Six years after treatment, $55 \%$ of the patients appeared to no longer have an eating disorder. They still displayed many of the symptoms of eating disorders, according to the authors, which is substantiated by the findings that the weight of the patients — despite marked improvement — was still much lower than normal. Six percent of the patients had died. Summarized, the results of such multimodal and total treatment are mediocre in the long run despite the fact that neither cost nor effort were spared.

The tendency is to think that a really complicated disorder was involved in most of these cases 
or that the patients were extraordinarily difficult. Eating disorders are virtually untreatable, and people basically have the disorder for life. Before accepting these views, it should be considered whether an absence of adequate treatment methods can also play a role in the limited success of the treatment of anorexia nervosa to date. One can lose a soccer match because the ball was too slippery, the grass was too long, or the goalposts were crooked; but the loss can also be due to a simple lack of good players.

The treatment that the Germans described is also common in the Netherlands. It is an allencompassing and expensive treatment, but its effectiveness is not at all clear: The percentage of patients who recover spontaneously - for example, within the space of six years — is simply not known. Those aspects of the treatment to which the modest degree of success should be attributed is also not clear: Which components should have an effect for what reason? It is also important to ask which aspects of the treatment may be counter-productive. What exactly happens, for example, during the confrontation with one's own body in the mirror? Does this really lead to a decline in the loathing of one's own body or actually intensify it? Is it a form of exposure or are cognitions restructured? Which cognitions and how exactly? It is a rhetorical question to ask whether it may not be particularly useful to first specify study, in the interest of the patients, the exact effects of such radical manipulations studied in isolation, e.g. in the behavioural laboratory, in with analogue groups, rather than applying the method without any further knowledge of the consequences for the patients.

Fortunately, there is some progress. Fairburn, Shafran, and Cooper (1999) recently published a cognitive behavioural account of anorexia nervosa. The authors formulated a model of the maintenance of anorexia nervosa that leads to clear and testable predictions about interventions and mechanisms of change. Models like this, and of course research testing the theory and intervention effect, are indispensable and a prerequisite for coming to effective treatment strategies.

\section{Treatment of bulimia nervosa}

Some twenty years ago, people also thought that Bulimia Nervosa was untreatable. In the years thereafter, the development of both etiological models and treatment protocols has accelerated enormously (e.g., Fairburn, 1981; Fairburn, Marcus, \& Wilson, 1993). As a consequence, some two-thirds of the patients with Bulimia Nervosa are cured after short-term treatment. At least 20 well-controlled and large-scale studies from a number of different countries and different research groups have unequivocally shown that cognitive behaviour therapy is currently the most effective form of treatment for Bulimia Nervosa (Agras, Walsh, Fairburn, Wilson, \& Kraemer, 2000; Whittal, Agras, \& Gould, 1999; Wilson 1996, 1999). Cognitive behaviour therapy is aimed at the modification of both the dysfunctional patterns of thinking and the disturbed eating behavior. On average, cognitive behaviour therapy leads to an $80 \%$ reduction of the eating binges and approximately $50 \%$ of the patients have no more eating binges whatsoever (Agras et al., 2000; Wilson 1996, 1999). Cognitions regarding eating behaviour, body shape, and body weight are less dysfunctional after the completion of the cognitive behaviour therapy and the pattern of eating is also more normal. The self-esteem of most patients increases; depressions clear up; personality disorders interfere less with daily life; and social functioning improves (Wilson, Fairburn, \& Agras, 1997). In short, Bulimia Nervosa no longer constitutes a problem for those providing treatment. 
This is, of course, not completely the case. While a marked reduction in eating binges and high abstinence percentages are obtained, it nevertheless appears that $37 \%$ of the patients still have an eating disorder six years following treatment: $20 \%$ still met the diagnostic criteria for Bulimia Nervosa and 17\% were diagnosed with an eating disorder NOS (Fairburn et al., 1995). In other words, six years following receipt of cognitive behaviour therapy approximately four of the six patients are cured, one still has Bulimia Nervosa, and one has a somewhat less serious eating disorder. Despite the latter, less rose-coloured figures, it is undeniably shown that cognitive behaviour therapy is to be the best that we can offer to people with Bulimia Nervosa. There is currently no better therapy available, which means that cognitive behaviour therapy should be the standard method of treatment for patients with Bulimia Nervosa. But researchers must ask themselves: How can the effectiveness of the treatment be expanded? There is considerable room for improvement.

An immediate measure might be to make the treatment more complicated and longer for severe patients in particular, but there is no empirical motivation for thinking that this is the best strategy. It has not been demonstrated that long-term treatment of certain patients is better than short and more specific treatment. There is also no explanation for the failure of some patients to benefit from cognitive behaviour therapy: predictors of treatment failure have yet to be found. What we do know is that the success of a treatment does not depend on the length or severity of the disorder (Schoemaker, 1998; Reas, Schoemaker, Zipfel, \& Williamson, 2001; Wilson, 1996). Perhaps we should therefore first identify what we need to treat and then think up the specific treatment.

It should be noted in this light that the usual cognitive behaviour therapy for Bulimia Nervosa consists of a large number of treatment components - many more than the term cognitive behaviour therapy suggests. The original cognitive behaviour therapy, as described and developed by Fairburn (see, for example, Fairburn et al., 1993) appears, in fact, to be a broad-spectrum treatment method but very well-defined and based on concrete protocol. It is a mix of techniques: diaries are kept, self-control techniques are taught, psycho-education is provided, exposure may be used, diet management is practiced, cognitive restructuring is attempted, problem-solving skills are trained, coping strategies are presented, and relapse prevention is undertaken. All of this occurs in a brief period of time: the treatment typically involves 19 sessions of one hour and the patients thus have a lot to do. Just as for the treatment of Anorexia Nervosa, however, we simply do not know which components of the multifaceted program are effective, necessary, sufficient, redundant, or possibly counter-productive.

Which of the techniques used during cognitive behaviour therapy appear to follow compellingly from the etiological models of the disorder? In fact, only cognitive restructuring and diet management. Cognitive restructuring is a good intervention for tackling the cognitive processes related to the loathing of one's body. Diet management can be used to tackle the problem of dieting, but it is also a procedure that decreases the classically conditioned urge to eat as the binge foods are actually included in the regular diets of the patients (see Jansen, 1998). It is possible that the effectiveness of the treatment is due to only one of these two interventions. In close collaboration of the present author with the eating team from the Mental Health Center Maastricht, this idea was tested. Patients with Bulimia Nervosa were randomly assigned to one of the following shortterm pure outpatient treatment conditions: cognitive therapy, diet management, or cue exposure with response prevention. The treatment for each patient took a total of 15 hours, and each patient was treated using only one technique. It should be noted that our patient group was just as seri- 
ously disturbed as the group of patients from the psychiatric clinic in Oxford. Here, tentative results for 41 patients with Bulimia Nervosa are given. The findings are tentative because a sufficient number of patients has yet to be examined and all of the data has not as yet been analyzed. Given that our sample is still quite small, that the 41 patients are distributed across three conditions, and that the results for the different conditions do not appear to differ, the discussion is limited to the group as a whole. At follow-up, one year after short-term therapy, the number of eating binges had decreased by $87 \%$. Moreover, $61 \%$ of the patients were free of eating binges. Not only has their eating behaviour improved but also their cognitions, mood, selfesteem, and social functioning (Jansen, 2001b).

What do these tentative findings suggest? They suggest that a therapy with numerous elements is not really necessary and that a single effective technique derived from a parsimonious model of the mechanisms maintaining the eating disorder is sufficient. Comparable results are obtained with short and simple treatment as with longer and more encompassing therapy. It should nevertheless be noted that the models of the maintenance mechanisms can still be greatly improved. It would not surprise if an even more valid model leads to even more parsimonious and effective interventions.

Everything considered, one clear conclusion can be drawn: an understandable reaction to a complicated problem is to apply a complicated treatment solution. Contrary to expectation, however, long and all-embracing therapy does not appear to be most effective for the treatment of difficult problems. Parsimonious models of the maintenance mechanisms, rather, dictate very specific interventions as not only necessary but also sufficient for tackling the maintenance mechanisms. A few tentative findings also suggest this to be the case.

\section{The daily practice}

In daily practice, various forms of treatment are employed for people with eating disorders from psychoanalysis to multimodal total packages. Recent American research (Mussell et al., 2000) shows that only one-third (!) of treatment providers treating patients with Bulimia Nervosa report using some form of cognitive behaviour therapy even though data of well-controlled empirical studies show this to currently be the preferred form of treatment for Bulimia Nervosa. Most of the psychotherapists questioned use "eclectic methods," which is often an euphemism for "doing what you feel like."

The question at this point is why patients with Bulimia Nervosa are not treated with cognitive behaviour therapy in the daily clinical practice? Why do empirically validated treatments enter daily clinical practice so slowly? Different reasons for this can be put forward (Persons, 1995).

A first reason is that psychotherapists receive much too little training on effective methods and far too much training on methods that appear to be ineffective. The American Psychological Association's Task Force for the distribution and promotion of psychological techniques analyzed the 1993 undergraduate programs for Clinical Psychology students and found attention to be paid to evidence-based treatments in less than half (46\%) of the training programs. APA accredited programs that devoted absolutely no attention to the training of empirically validated treatments were also encountered. Of the American psychotherapists who treat Bulimia Nervosa, one-third utilize cognitive behaviour therapy and only $65 \%$ of those who report using cognitive behaviour 
therapy ever received training on this form of treatment! The Task Force found that between $20 \%$ and $74 \%$ of the training and supervision programs also addressed completely ineffective treatment methods. In short: Clinical psychologists are poorly educated. Therapists are trained on therapies that are known to be ineffective but not on therapies that have clearly been shown to be effective.

The quality of the psychotherapy training programs should be better monitored and thereby only training on the empirically validated treatment methods for a particular disorder provided when known. Obviously the APA and her Dutch sister (Netherlands Institute of Psychologists; NIP) should only accredit those forms of treatment that have been empirically validated. And as the field of clinical practice develops, psychotherapists should be required and given enough time to train or regularly refresh themselves and others on empirically validated treatment techniques. The Dutch Ministry of Health, Welfare and Sport would have been well-advised to only nominate hospital units as specialised in the treatment of eating disorders when their treatment is evidencebased, i.e. when they are treating eating disordered patients with cognitive behavior therapy. In fact, the units are not at all selected on their treatment quality or the use of evidence-based treatments. They were selected on interest, regional staggering and treatment capacity.

The fact that psychotherapists generally do not read about the results of outcome studies published in specialized journals and are often completely unaware of the empirical status of their favorite treatment is understandable. Their entire week is fully booked with patient contacts and team meetings. It would be better if keeping up with the literature in the field, conference attendance, and the teaching or following of training workshops also came to be considered part of the psychotherapist's task.

Researchers should also expend more effort to disseminate important findings with regard to effective treatments in an accessible form. The pharmaceutical industry spends millions on the distribution of information about newly developed pills while psychologists do virtually nothing with regard to newly developed and clearly effective treatment protocols (Barlow, 1994, in Persons, 1995). There are different journals and book series that consider the publication of readable protocols, manuals, and important research results to be of paramount importance. Such publications should certainly be given greater prestige but the readers should also be able to count on the high standards of the publications: that is, the information presented in the publications should indeed be empirically valid.

A last important reason for why actual clinical practice pays so little attention to demonstrated treatment effectiveness is that many psychotherapists tend to be very skeptical and often think that they are treating a different type of patient than the researcher. The criticism put forth by some therapists is that researchers select their patients and thus end up with the least severe cases while the really most severe cases populate clinical practice. And a treatment protocol is much too simple for such severe cases. For more difficult patients, it is absolutely necessary that the treatment be adapted as much as possible to the individual client - or so they say. In a very elegant study, Schulte, Kunzel, Pepping, and Schulte-Badenberg (1992) explicitly tested the hypothesis that an individually tailored therapy (i.e., therapy adapted to the individual) is better than therapy based on a general treatment protocol. A total of 120 people with an anxiety disorder were treated by 28 experienced therapists. The experimental group $(n=40)$ received individually tailored therapy: the treatment was based on the individual problem analysis of the patient and the (experienced) therapist could make use of any and every technique considered good for the patient. The first control group ( $n=40)$ was treated according to the protocol. The therapy was 
described in a manual with clear instructions regarding what should be done (exposure in vivo and cognitive restructuring) and when it should be done. The treatment was the same for all of the patients in this group and thus independent of any individual peculiarities. The second control group or the "yoked control group" received the therapy tailored to a patient in the experimental group. Each patient in the yoked control group thus received the same treatment as a randomly selected individual from the experimental group: The treatment in the yoked control group was thus individually tailored to another person.

Following the protocol treatment, $67 \%$ of the patients appeared to be completely or largely cured; following the individually tailored therapy, this was 39\%; and following receipt of tailored therapy yoked to a different individual, $44 \%$ of the patients were completely or largely cured. Given that the cure percentages for the two "individually tailored groups" were more or less equal, it can be concluded that the individual tailoring of the therapy is not of importance for the success of the therapy; whether someone is treated according to his own problem analysis or that of the neighbor makes no difference. The exposure protocol appeared to be best and exposure was also, incidentally, the best predictor of the success of the individually tailored therapy (some of the experienced therapists considered exposure to be indicated for the individually tailored treatment of some patients).

The study involved representative patients and experienced therapists who were free to do as they saw fit. Nevertheless, the therapies that the therapists intuitively judged to be most appropriate were less successful than the the standardized exposure protocol, derived from a model on the mechanism maintaining anxiety disorders. There is no reason to think that these findings are not generalizable to the treatment of eating disorders.

\section{Can eating disorders be prevented?}

Experts in the field and employees at the Dutch Ministry of Health, Welfare and Sport also reason that eating disorders should be detected as quickly as possible so that patients can be treated as early as possible and thereby have a greater chance of cure. This sounds very plausible, but is it really the case?

In an excellent argument, Schoemaker (1998) showed that secondary prevention of eating disorders is of little use for a number of reasons. First, eating disorders involve small numbers of patients. Precisely because the prevalence is so low and because we know very little about the risk factors, extremely large population groups must be screened to discover a few cases. But this is not the greatest problem. Prevention requires the early detection of potential cases while this is actually the crux of the matter when it comes to eating disorders: those at risk appear to massively hide from screening research. Different studies have shown a high percentage of those who decide against further participation in research to suffer from an eating disorder. The target individuals either deny being sick or are so ashamed of their condition that they refuse to participate further. The cases that stand out during screening and prove approachable are usually already undergoing treatment or refuse any treatment. In these cases, screening thus contributes nothing more than when the disorder is spontaneously reported and subsequently treated. Early detection of cases is labor-intensive, difficult, and expensive. This is nevertheless not a reason for not doing it. A clear reason for not attempting to detect cases of disorder as early as possible is that it has 
never been proven that the early treatment of Anorexia Nervosa and Bulimia Nervosa produces better treatment results than intervention at a later point (Schoemaker, 1998; Reas et al., 2001).

When it is tried to be one step ahead of the eating disorder, one speaks of primary prevention. With the circulation of information to groups at risk, it is attempted to prevent the occurrence of an eating disorder. Educational programs for schools have been developed in a variety of countries. This means that lessons are devoted during both elementary school and high school to the thinness ideal in western culture and the influence of such on women; puberty and the physical changes occurring at this time; dieting and losing weight; the characteristics of eating disorders, the consequences of eating disorders, possibilities for treatment, etc. The assumption on the part of prevention workers is that knowledge of the aforementioned topics will reduce the tendency to diet and thereby the prevalence of eating disorders among the target group. Such a program is also being used at 1250 Dutch schools (Noordenbos, 1999). The effects of this program have never been documented, however. A recent study from England suggests that such programs can occasionally work counter-productively. In the relevant study, it was determined that knowledge of dieting and eating disorders indeed increased following intervention and that the incidence of dieting behaviour initially declined. But follow-up at six months showed the incidence of dieting behaviour to have increased considerably. In fact, those who had followed the program were found to diet more than those who had not followed the program and also worried more about their weight (Carter, Stewart, Dunn, \& Fairburn, 1997). Knowledge need not necessarily protect. Quite to the contrary, prevention programs can be counter-productive. It is really quite crazy that no empirical research is conducted into the effects of such prevention programs prior to their large-scale implementation. Making such research obligatory would therefore be a good prevention measure.

\section{The current policy of the Dutch Ministry}

Coming back to the current policy of the Dutch Ministry of Health, Welfare, and Sport. It is expected that, through the mere nomination of hospital units specialized in the treatment of eating disorders by the Ministry, the waiting lists will decrease and treatment will be more effective. It is, however, not necessarily true that the mere selection of some hospital units will increase the supply of effective treatment. It might be predicted that the supply will only get smaller in the end. For what is now the case? Knowing a hospital unit that is supposed to be specialized in the treatment of eating disorders, therapists at local Mental Health Centers tend to consider themselves insufficiently qualified for the treatment of eating disorders. (Which is interesting, because most therapists in the specialised units are not better trained in empirically validated eating disorder therapies than therapists outside these units). The presence of a specialized unit is supposed to guarantee good treatment by specialized therapists and the Mental Health Center therapists therefore refer every patient as quickly as possible to a specialized unit in the area. That the waiting lists in the selected hospitals are increasing and insufficient treatment capacity arises as a result is beyond a doubt.

It was already suggested that the continued training of psychotherapists at Mental Health Centers and other outpatient psychotherapeutic clinics is of critical importance. The Minister obviously should have invested lots of funds in a broad array of workshops, training programs, edu- 
cational programs, and supervision programs in the area of cognitive behaviour therapy for eating disorder therapists instead of the mere and easy assignment of the label "specialised" to some hospital units. With the training of a large number of psychotherapists in cognitive behavior therapy for eating disorders, the treatment supply would have been actually multiplied and far more effective.

In addition to this, many more of the women with eating disorders standing at the gate of a specialized hospital unit tend to be hospitalized than those reporting to a non-clinical institution. This is indicated by the findings that the Steering Group on Eating Disorders in the Netherlands (SEN, 1998) presents in its report. It is reported that specialized hospital units treat $26 \%$ of the patients with Bulimia Nervosa on a completely inpatient basis and $30 \%$ on a daily inpatient. Only $40 \%$ are treated on an outpatient basis (SEN, 1998). When we compare these figures to those for the university psychiatric clinic in Oxford and those for the academic Mental Health Center in Maastricht, a very different picture emerges. In both places, more than $95 \%$ of the patients with Bulimia Nervosa are given short-term cognitive behavior therapy consisting of less than 20 contact hours. The $95 \%$ of the patients treated on such an outpatient basis in Oxford and Maastricht is almost 2.5 times more than the $40 \%$ in the specialized clinics. The specialized hospital units have not shown their treatment to be more effective or their patients to be more seriously ill than those in Oxford or Maastricht. A similar picture arises for the Not Otherwise Specified cases of eating disorders including the Binge Eating Disorder. It might be clear that less hospitalization of patients with Bulimia Nervosa and Binge Eating Disorder leaves more room for the inpatient treatment of really severe Anorexia Nervosa patients. In other words, the specialization of hospital units appears to create a tendency for the providers of outpatient care to simply refer and a tendency for the providers of inpatient care to hospitalize; thereby ending up with significantly higher hospitalization rates. Both developments suggest a shortage of treatment capacity, which is an artifact. Psychotherapists outside the specialized units are capable of treating a large percentage of the eating disordered patients they see. When they all are trained in cognitive behavior therapy and treat most of the eating disorders accordingly, treatment all-over will be more effective, unnecessary hospitalization will decrease and a greater number of beds for the severe cases will remain.

\section{Conclusions}

In daily practice, only one-third of the psychotherapists treating patients with Bulimia Nervosa report using some form of cognitive behaviour therapy even though data of well-controlled empirical studies show this to currently be the preferred form of treatment for Bulimia Nervosa. Of these psychotherapists doing cognitive behavior therapy only 65\% ever received training on this form of treatment. The Dutch Ministry of Health, Welfare and Sport wants to improve treatment for patients with eating disorders. Therefore, she nominated a number of general hospital units in the country as specialized in the treatment of eating disorders. However, the practise of evidence-based psychotherapy, i.e. cognitive behaviour therapy, was not a criterion for selection of these units.

It was argued that a mere nomination of several hospital units as being "specialized centers" in the treatment of eating disorders will not lead to a decrease of the waiting lists and an increase 
of effective treatment supply. The nomination of the centers invites others to refer their patients to these centers, which means a decreased treatment supply for eating disordered patients in the numerous local Mental Health Centers. Moreover, the Dutch Ministerial action stimulates undesirable hospitalization in the specialized units. The Minister obviously should have done two other things to improve the amount and quality of treatment supply for eating disorders. First, she should have invested in a broad array of workshops, training and supervision programs in cognitive behaviour therapy for psychotherapists working with eating disorders. Second, since nothing is as practical as a good theory, she should have invested in more strategic or reductionistic experimental research which is aimed at identifying the real factors that cause or maintain the disorders. We still do not know enough about the mechanisms that maintain an eating disorder and that precisely is the reason for why treatment of eating disorders (Anorexia Nervosa in particular) is not as effective as it should be. The facilitation of research into parsimonious models of the relevant mechanisms and experimental testing of these mechanisms could have been a very effective move to stimulate more directed and effective treatment than is currently the case.

The Dutch Ministry thus placed the horse behind the cart rather than in front of it. Investment of all available funds in the (assumed) expansion of treatment availability is not very efficient when there are still no effective treatments. The traffic jam behind the cart can only get longer as new patients line up (i.e. longer waiting lists). And although the possibility of the horse still being able to get ahead cannot be ruled out, the manner of working is not very handy and the flow of traffic will never be particularly smooth. A good theory of the mechanisms maintaining eating disorders is needed. When we understand how eating disorders arise or why they continue to exist, effective treatments will be more or less apparent.

\section{Acknowledgements}

Thanks are expressed to Marcel van den Hout for the rich discussions we had through the years.

\section{References}

Agras, W. S., Walsh, T., Fairburn, C. G., Wilson, G. T., \& Kraemer, H. C. (2000). A multicenter comparison of cognitive-behavioral therapy and interpersonal psychotherapy for bulimia nervosa. Archives of General Psychiatry, 57, 459-466.

Bloks, J., van Furth, E., \& Hoek, H. (1999). Behandelingsstrategieen bij anorexia nervosa (Treatment strategies of anorexia nerosa). Houten/Diegem: Bohn Stafleu van Loghum.

Brownell, K. D., \& Fairburn, C. G. (1995). Eating disorders and obesity. A comprehensive handbook. New York: Guilford Press.

Carter, J. C., Stewart, A., Dunn, V., \& Fairburn, C. G. (1997). Primary prevention of eating disorders: might it do more harm than good? International Journal of Eating Disorders, 22, 167-172.

De Groot, A. D. (1961). Methodologie. Grondslagen van onderzoek en denken in de gedragswetenschappen. (Methodology. Foundations of research and thinking in the behavioural sciences). Den Haag: Mouton.

Fairburn, C. G. (1981). A cognitive behavioural approach to the management of bulimia. Psychological Medicine, 11, $707-711$.

Fairburn, C. G., Marcus, M. D., \& Wilson, G. T. (1993). Cognitive-behavioural therapy for binge eating and bulimia 
nervosa: A comprehensive treatment manual. In C. G. Fairburn, \& G. T. Wilson, Binge eating: nature, assessment and treatment (pp. 361-404). The Guilford Press.

Fairburn, C. G., Norman, P. A., Welch, S. L., O’Connor, M. E., Doll, H. A., \& Peveler, R. C. (1995). A prospective study of outcome in bulimia nervosa and the long term effects of three psycological treatments. Archives of General Psychiatry, 52, 304-312.

Fairburn, C. G., Welch, S. L., Doll, H. A., Davies, B. A., \& O’Connor, M. E. (1997). Risk factors for bulimia nervosa. Archives of General Psychiatry, 54, 509-517.

Fairburn, C. G., Shafran, R., \& Cooper, Z. (1999). A cognitive behavioural theory of anorexia nervosa. Behaviour Research and Therapy, 37, 1-13.

Fichter, M. M., \& Quadflieg, N. (1999). Six-year course and outcome of anorexia nervosa. International Journal of Eating Disorders, 26, 359-385.

Garfinkel, P. E., \& Garner, D. M. (1982). Anorexia Nervosa. A Multidimensional Perspective. In The Multidetermined Nature of Anorexia Nervosa (p. 188-213). New York: Brunner/Mazel.

Garner, D. M. (1997). Psychoeducational principles in treatment. In D. M. Garner, \& P. E. Garfinkel, Handbook of treatment for eating disorders (pp. 145-178). The Guilford Press.

Jansen, A. (1998). A learning model of binge eating: cue reactivity and cue exposure. Behaviour Research and Therapy, $36,257-272$.

Jansen, A. (2001a). Ik zie, ik zie wat jij ook ziet: over het lichaamsbeeld van vrouwen met eetstoornissen (I see what you see: about the body image of women with eating disorders). Gedrag and Gezondheid (Behavior and Health), 29, 25-34.

Jansen, A. (2001b). Cue exposure vs cognitive therapy vs diet management in the treatment of bulimia nervosa (in preparation).

Jansen, A., \& Boon, L. (1991). Eetstoornissen en multicausaliteit. (Eating disorders and multicausality). De Psycholoog (The Psychologist), 7 (8), 325-330.

Mussell, M., Crosby, R., Crow, S., Knopke, A., Peterson, C., Wonderlich, S., \& Mitchell, J. (2000). Utilization of empirically supported psychotherapy treatments for individuals with eating disorders: a survey of psychologists. International Journal of Eating Disorders, 27, 230-237.

Noordenbos, G. (1999). Preventie van eetstoornissen. (Prevention of eating disorders). In J. Bloks, E. van Furth, \& H. Hoek, Behandelingsstrategieen bij anorexia nervosa (Treatment strategies of anorexia nerosa) (pp. 105-112). Houten/Diegem: Bohn Stafleu van Loghum.

Persons, J. (1995). Why practicing psychologists are slow to adopt empirically-validated treatments. In S. C. Hayes, V. M. Follette, R. M. Dawes, \& K. E. Grady, Scientific standards of psychological practice: issues and recommendations (pp. 141-158). Reno: Context Press.

Reas, D. L., Schoemaker, C., Zipfel, S., \& Williamson, D. A. (2001). The prognostic value of duration of illness and early intervention in bulimia nervosa: a systematic review of outcome literature. International Journal of Eating Disorders (in press).

Schoemaker, C. (1998). The principles of screening for eating disorders. In W. Vandereycken, \& G. Noordenbos, The prevention of eating disorders. Studies in eating disorders, an international series (pp. 187-213). London: The Atlene Press.

Schulte, D., Kunzel, R., Pepping, G., \& Schulte-Badenberg, T. (1992). Tailor-made versus standardized therapy of phobic patients. Advanced Behaviour Research and Therapy, 14, 67-92.

Schwartz, D. J., Phares, V., Tantleff-Dunn, S., \& Thompson, J. K. (1999). Body image, psychological functioning, and parental feedback regarding physical appearance. International Journal of Eating Disorders, 25, 339-343.

Stice, E., Mazotti, L., Krebs, M., \& Martin, S. (1998). Predictors of adolescent dieting behaviours: a longitudinal study. Psychology of Addictive Behaviours, 12, 195-205.

Steering Group on Eating Disorders in the Netherlands (SEN, 1998). Final report (in Dutch).

Van Hoeken, D., \& Hoek, H. W. (1999). Epidemiologie (Epidemiology). In J. Bloks, E. van Furth, \& H. Hoek, Behandelingsstrategieen bij anorexia nervosa (Treatment strategies of anorexia nerosa). Houten/Diegem: Bohn Stafleu van Loghum.

Vanderlinden, J. (2000). Anorexia Nervosa overwinnen. (Overcome anorexia nervosa). Tielt: Lannoo.

Whittal, M. L., Agras, W. S., \& Gould, R. A. (1999). Bulimia Nervosa: a meta-analysis of psychosocial and pharmacological treatments. Behaviour Therapy, 30, 117-135. 
Wilson, G. T. (1996). Treatment of bulimia nervosa: when CBT fails. Behaviour Research and Therapy, 34, 197-212. Wilson, G. T. (1999). Cognitive behaviour therapy for eating disorders: progress and problems. Behaviour Research and Therapy, 37, S79-S95.

Wilson, G. T., Fairburn, G. C., \& Agras, W. S. (1997). Cognitive-behavioural therapy for bulimia nervosa. In D. M. Garner, \& P. E. Garfinkel, Handbook of treatment for eating disorders. New York: Guilford Press. 